\title{
On bipartite pure-state entanglement structure in terms of disentanglement
}

\author{
Fedor Herbut (E-mail: fedorh@infosky.net and fedorh@mi.sanu.ac.yu) \\ Serbian Academy of Sciences and Arts, Knez Mihajlova 35, \\ 11000 Belgrade, Serbia
}

Schrödinger's disentanglement [E. Schrödinger, Proc. Cambridge Phil. Soc. 31, 555 (1935)], i. e., remote state decomposition, as a physical way to study entanglement, is carried one step further with respect to previous work in investigating the qualitative side of entanglement in any bipartite state vector. Remote measurement (or, equivalently, remote orthogonal state decomposition) from previous work is generalized to remote linearly-independent complete state decomposition both in the non-selective and the selective versions. The results are displayed in terms of commutative square diagrams, which show the power and beauty of the physical meaning of the (antiunitary) correlation operator inherent in the given bipartite state vector. This operator, together with the subsystem states (reduced density operators), constitutes the so-called correlated subsystem picture. It is the central part of the antilinear representation of a bipartite state vector, and it is a kind of core of its entanglement structure. The generalization of previously elaborated disentanglement expounded in this article is a synthesis of the antilinear represen- 
tation of bipartite state vectors, which is reviewed, and the relevant results of Cassinelli et al. [J. Math. Analys. and Appl., 210, 472 (1997)] in mathematical analysis, which are summed up. Linearly-independent bases (finite or infinite) are shown to be almost as useful in some quantum mechanical studies as orthonormal ones. Finally, it is shown that linearly-independent remote pure-state preparation carries the highest probability of occurrence. This singles out linearly-independent remote influence from all possible ones. 


\section{INTRODUCTION}

There are different measures of the amount of entanglement in bipartite states. In pure states they all coincide. Hence, this is well understood. But one may wonder the measure of what is at issue; i. e., what is the structure of entanglement, or what is its qualitative side.

According to Schrödinger, the natural way to investigate entanglement is to perform disentanglement: ${ }^{1}$ It consists in measurements on the nearby subsystem. Since it is simultaneously a measurement on the composite system, the bipartite state becomes a mixed one. As a consequence, one has an actual decomposition (as opposed to a potential or mathematical one) of the remote subsystem state.

In previous work, ${ }^{2}$ complete remote measurement or, equivalently, complete remote orthogonal state decomposition, was studied as a first step in carrying out Schrödinger's program, and the concept of twin observables was introduced. They gave physical meaning to the so-called correlated subsystem picture. ${ }^{3}$

Mathematically, the optimal way to study pure-state bipartite entanglement is to use the antilinear operator representation of the state vector. As it is well known, in theoretical physics mathematics is inextricably connected with physics. In the mentioned previous work it turned out that the antiunitary polar factor, the so-called correlation operator, plays a central role in establishing the concepts of twin observables and remote measurement. Naturally, this operator is endowed with basic physical meaning.

The antilinear operator representation of bipartite state vectors and the polar factorizations of these operators are summed up and shortly reviewed in Section 2. Delving into the antilinear approach may require some effort on part of some readers, but it is pure-state bipartite entanglement and not this 
author who made it optimal. Eventually, the insight gained should make it worth the effort.

In this article the physical content of the correlated subsystem picture is extended one step beyond remote measurement.

The organization of the rest of the article goes as follows. In section 3 the relevant purely mathematical results on classification of all linearlyindependent complete decompositions of any given density operator (that with an infinite-dimensional range included $)^{4}$ are shortly stated. Besides, they are, to some extent, elaborated in order to show that linearly-independent bases can be almost as useful as orthonormal ones (to encourage their use at least in entanglement studies in quantum mechanics ). In section 4 the first result of this paper, the generalized twin observables, consisting of twin observables and of extended twin observables, are presented in the form of Theorem 1 and a commutative square diagram. In section 5 selective (or specific-result) nearby-subsystem measurement that gives rise to so-called remote pure-state preparation is payed special attention to in terms of Theorem 2 and another square commutative diagram. Besides, in Theorem 3 the physical meaning of linearly-independent remote pure-state preparation is clarified. In section 6 concluding remarks point out the essential features of the results.

As a technical remark, it should be noted that by a basis (without further specification) in a subspace is meant a complete orthonormal set, i. e., one spanning the subspace. We will also deal with linearly-independent bases in linear manifolds (cf Corollaries 1 and 3).

\section{THE CORRELATED SUBSYSTEM PICTURE}

The correlated subsystem picture is based on the role of the antiunitary 
correlation operator $U_{a}$ inherent in any bipartite state vector $|\Phi\rangle_{12}$. The correlation operator is the antiunitary polar factor of the antilinear HilbertSchmidt operator $A_{a}$ that maps the state space of subsystem 1 into that of subsystem 2. Such an operator, in turn, gives an antilinear representation of any given bipartite state vector $|\Phi\rangle_{12}$.

Antilinear operators were introduced in physics from the mathematical literature $^{5}$ by Jauch. ${ }^{6}$ They were utilized in Ref. 7 and in the first-step bipartite pure-state studies. ${ }^{2,8}$ The main result of these was establishing the correlated subsystem picture of $|\Phi\rangle_{12}$ (with the twin observables) as a core of its structure.

\section{A. The mathematical part}

Let $|\Phi\rangle_{12}$ be a given state vector of an arbitrary bipartite pure state with a nearby (1) and a remote (2) subsystem. Naturally, $|\Phi\rangle_{12} \in\left(\mathcal{H}_{1} \otimes \mathcal{H}_{2}\right)$, where the tensor factors are complex separable Hilbert spaces.

The first notion that is being utilized is that of the partial scalar product: If $|\psi\rangle_{1}$ is an arbitrary vector of the nearby subsystem, then the partial scalar product

$$
\left\langle\left.\psi\right|_{1} \mid \Phi\right\rangle_{12} \in \mathcal{H}_{2}
$$

gives a vector in the state space $\mathcal{H}_{2}$ of the remote subsystem. It can be defined and evaluated by introducing bases $\left\{|j\rangle_{1}: \forall j\right\} \subset \mathcal{H}_{1}$ and $\left\{|k\rangle_{2}: \forall k\right\} \subset \mathcal{H}_{2}$ and expanding $|\Phi\rangle_{12}$ in them:

$$
|\Phi\rangle_{12}=\sum_{j} \sum_{k} f_{j k}|j\rangle_{1}|k\rangle_{2}
$$

Then $\left\langle\left.\psi\right|_{1} \mid \Phi\right\rangle_{12}$ is obtained in terms of the ordinary scalar product in $\mathcal{H}_{1}$ :

$$
\left\langle\left.\psi\right|_{1} \mid \Phi\right\rangle_{12}=\sum_{j} \sum_{k}\left[f_{j k}\left(\left\langle\left.\psi\right|_{1} \mid j\right\rangle_{1}\right)\right]|k\rangle_{2}
$$


The point is, of course, that, as it is straightforward to show, the rhs is always defined (in case of infinite sums, one has convergence), and the lhs is independent of the choice of the subsystem bases, and thus a well-defined element of $\mathcal{H}_{2}$.

The next notion is that of the antilinear operator representation of a bipartite state vector $|\Phi\rangle_{12}$ : Relation (1) is actually an antilinear, i. e., expansion-coefficients complex-conjugating, map $A_{a}$ of the entire space $\mathcal{H}_{1}$ into $\mathcal{H}_{2}$ :

$$
\left(A_{a}|\psi\rangle_{1}\right)_{2} \equiv\left\langle\left.\psi\right|_{1} \mid \Phi\right\rangle_{12} \in \mathcal{H}_{2} .
$$

The operator $A_{a}$ defines its adjoint $A_{a}^{\dagger}$, which maps in the antilinear way $\mathcal{H}_{2}$ into $\mathcal{H}_{1}$. This is done via the pair of scalar products, that in $\mathcal{H}_{1}$ and that in $\mathcal{H}_{2}$ :

$$
\forall \psi_{1} \in \mathcal{H}_{1}, \quad \forall \chi_{2} \in \mathcal{H}_{2}: \quad\left(\chi_{2},\left(A_{a} \psi_{1}\right)_{2}\right)_{2}=\left(\left(A_{a}^{\dagger} \chi_{2}\right)_{1}, \psi_{1}\right)_{1}^{*},
$$

where the asterisk denotes complex conjugation. It is easy to see that (4) defines adjoining as a linear operation.

The operators $A_{a}$ and $A_{a}^{\dagger}$ are called Hilbert-Schmidt ones because

$$
\operatorname{tr}\left(A_{a}^{\dagger} A_{a}\right)_{1}<\infty, \quad \operatorname{tr}\left(A A_{a}^{\dagger}\right)_{2}<\infty .
$$

The set of all antilinear Hilbert-Schmidt operators mapping $\mathcal{H}_{1}$ into $\mathcal{H}_{2}$ is a complex separable Hilbert space, in which the scalar product is defined as

$$
\forall A_{a}, A_{a}^{\prime}: \quad\left(A_{a}, A_{a}^{\prime}\right) \equiv \operatorname{tr}\left(A_{a}^{\prime \dagger} A_{a}\right)_{1} .
$$

It is straightforward to show that (3) constitutes an isomorphism of the complex separable Hilbert space of all ordinary bipartite vectors $|\Phi\rangle_{12}$ onto that of all antilinear Hilbert-Schmidt operators mapping $\mathcal{H}_{1}$ into $\mathcal{H}_{2}$. 
In the sense of this isomorphism, one can speak of $A_{a}$ as the antilinear operator representative of $|\Phi\rangle_{12}$.

It is known that the reduced density operators $\rho_{1} \equiv \operatorname{tr}_{2}\left(|\Phi\rangle_{12}\left\langle\left.\Phi\right|_{12}\right)\right.$ and $\rho_{2} \equiv \operatorname{tr}_{1}\left(|\Phi\rangle_{12}\left\langle\left.\Phi\right|_{12}\right) \quad\right.$ of any given bipartite state vector $|\Phi\rangle_{12}$, which describe the respective subsystem states, have equal positive parts of their spectra, i. e., their positive eigenvalues, together with their multiplicities, coincide. Further, it is known that if one expands $|\Phi\rangle_{12}$ in any eigen-subbasis $\left\{\left|r_{i}\right\rangle_{1}: \forall i\right\} \quad$ of $\rho_{1}$ spanning its range, then one obtains the so-called biorthogonal Schmidt expansion

$$
|\Phi\rangle_{12}=\sum_{i} r_{i}^{1 / 2}\left|r_{i}\right\rangle_{1}\left|r_{i}\right\rangle_{2}
$$

where $\left\{r_{i}: \forall i\right\}$ are the positive eigenvalues of $\rho_{1}$ corresponding to its mentioned eigenvectors, and $\left\{\left|r_{i}\right\rangle_{2}: \forall i\right\}$ turn out necessarily to be eigenvectors of $\rho_{2}$ spanning its (equally dimensional) range. Actually, one can write the spectral forms as follows

$$
\rho_{1}=\sum_{i} r_{i}\left|r_{i}\right\rangle_{1}\left\langle\left. r_{i}\right|_{1}, \quad \rho_{2}=\sum_{i} r_{i} \mid r_{i}\right\rangle_{2}\left\langle\left. r_{i}\right|_{2} .\right.
$$

What the standard approach is lacking is any expression of the correlations between the two subsystems that the bipartite state implies. This is where the antilinear operator representation of the bipartite state has a marked advantage.

If one writes down the polar factorizations of $A_{a}$, one obtains

$$
A_{a}=U_{a} \rho_{1}^{1 / 2}, \quad A_{a}=\rho_{2}^{1 / 2} U_{a} Q_{1}
$$

where $U_{a}$ is the antilinear unitary (or antiunitary) correlation operator, which maps the (topologically) closed range $\overline{\mathcal{R}}\left(\rho_{1}\right)$ of $\rho_{1}$ onto $\overline{\mathcal{R}}\left(\rho_{2}\right)$, 
that of $\rho_{2}$ (preserving the scalar product up to complex conjugation). The Hermitian polar factors are the positive-operator roots of the corresponding reduced density operators, and $Q_{1}$ is the range-projector of $\rho_{1}$. The operator $U_{a}$ is uniquely determined by $A_{a}$ (i. e., by $|\Phi\rangle_{12}$ ), e. g., by $U_{a} Q_{1}=\tilde{\rho}_{2}^{-1 / 2} A_{a} \quad$ (as follows from $(9 \mathrm{~b})$ ), where $\tilde{\rho}_{2}$ is the reducee of $\rho_{2}$ in $\overline{\mathcal{R}}\left(\rho_{2}\right)$. (See also remark beneath relation (12c).) (An elementary discussion of polar factorization of linear operators in one space is given in Ref. 9, and a more general one in Appendix 4 of Ref. 2. The polar factorizations (9a,b) of $A_{a}$ differ very little from this.)

It turns out that

$$
\rho_{2}=\left(U_{a} \rho_{1} U_{a}^{-1}\right)_{2} Q_{2}
$$

is valid, where $Q_{2}$ is the range-projector of $\rho_{2}$. Utilizing $U_{a}$, the above Schmidt expansion and the spectral forms can be rewritten as follows:

$$
|\Phi\rangle_{12}=\sum_{i} r_{i}^{1 / 2}\left|r_{i}\right\rangle_{1}\left(U_{a}\left|r_{i}\right\rangle_{1}\right)_{2}
$$

and

$$
\rho_{1}=\sum_{i} r_{i}\left|r_{i}\right\rangle_{1}\left\langle\left. r_{i}\right|_{1}, \quad \rho_{2}=\sum_{i} r_{i}\left(U_{a}\left|r_{i}\right\rangle_{1}\right)_{2}\left(\left\langle\left. r_{i}\right|_{1} U_{a}^{\dagger}\right)_{2}\right.\right.
$$

(Note that $U_{a}^{\dagger}=U_{a}^{-1}$.) Actually,

$$
\forall i: \quad\left|r_{i}\right\rangle_{2}=U_{a}\left|r_{i}\right\rangle_{1}
$$

Thus, the correlation operator $U_{a}$ can be read off from the Schmidt biorthogonal expansion (11) when the latter is explicitly evaluated.

If $\left\{|j\rangle_{1} ; \forall j\right\}$ is a basis in $\mathcal{H}_{1}$, one can uniquely expand the bipartite state, and, as easily seen, one obtains

$$
|\Phi\rangle_{12}=\sum_{j}|j\rangle_{1}\left(A_{a}|j\rangle_{1}\right)_{2}
$$


The antilinear representation $A_{a}$ of $|\Phi\rangle_{12}$ can be read off from this because the antilinear operator $A_{a}$ is continuous (cf Appendix 2 in Ref. 2 ); hence it is determined by its action on a basis.

Relation (13a) can also be understood as giving the inverse of isomorphism (3), i. e., as determining the map $A_{a} \rightarrow|\Phi\rangle_{12}$. (It is straightforward to show that the lhs of (13a) does not depend on the choice of the basis.)

If the basis in (13a) is an eigenbasis of $\rho_{1}$, then, and only then, as immediately seen from (9a), the general expansion (13a) takes on the special form of the biorthogonal Schmidt expansion (7).

The adjoint antilinear Hilbert-Schmidt operators $A_{a}^{\dagger}$ also form a complex separable Hilbert space in their turn with the scalar product

$$
\left(A_{a}^{\dagger},\left(A_{a}^{\dagger}\right)^{\prime}\right) \equiv \operatorname{tr}\left(A_{a}^{\prime} A_{a}^{\dagger}\right)_{2}
$$

They give the second antilinear operator representation for bipartite vectors via the isomorphism:

$$
\forall|\Phi\rangle_{12}: \rightarrow A_{a}^{\dagger}: \quad \forall|\chi\rangle_{2}:\left(A_{a}^{\dagger}|\chi\rangle_{2}\right)_{1} \equiv\left\langle\left.\chi\right|_{2} \mid \Phi\right\rangle_{12} \in \mathcal{H}_{1}
$$

Associating $A_{a}^{\dagger}$ with $A_{a}(\mathrm{cf}(4))$ is also an isomorphism. (Any two of the mentioned three isomorphisms of bipartite state spaces multiply, i. e., give, when taken one after the other, the third one.)

One has the following relations that are symmetric to (9a)-(13a) in terms of the adjoint antilinear operator representation of $|\Phi\rangle_{12}$ :

$$
A_{a}^{\dagger}=U_{a}^{-1} \rho_{2}^{1 / 2}, \quad A_{a}^{\dagger}=\rho_{1}^{1 / 2} U_{a}^{-1} Q_{2} .
$$

Further,

$$
\rho_{1}=U_{a}^{-1} \rho_{2} U_{a} Q_{1}
$$




$$
|\Phi\rangle_{12}=\sum_{i} r_{i}^{1 / 2}\left(U_{a}^{-1}\left|r_{i}\right\rangle_{2}\right)_{1}\left|r_{i}\right\rangle_{2}
$$

where $\left\{\left|r_{i}\right\rangle_{2}: \forall i\right\}$ is any eigen-sub-basis of $\rho_{2}$ spanning the range of the latter, and $\left\{r_{i}: \forall i\right\}$ are the corresponding (positive) eigenvalues.

$$
\begin{gathered}
\rho_{1}=\sum_{i} r_{i}\left(U_{a}^{-1}\left|r_{i}\right\rangle_{2}\right)_{1}\left(\left\langle\left. r_{i}\right|_{2} U_{a}\right)_{1}=\sum_{i} r_{i}\left(U_{a}^{-1}\left(\left|r_{i}\right\rangle_{2}\left\langle\left. r_{i}\right|_{2}\right) U_{a}\right)_{1},\right.\right. \\
\rho_{2}=\sum_{i} r_{i}\left|r_{i}\right\rangle_{2}\left\langle\left. r_{i}\right|_{2} .\right.
\end{gathered}
$$

In general,

$$
|\Phi\rangle_{12}=\sum_{k}\left(A_{a}^{\dagger}|k\rangle_{2}\right)_{1}|k\rangle_{2}
$$

where $\left\{|k\rangle_{2}: \forall k\right\}$ is any basis in $\mathcal{H}_{2}$. Again, it is clear from (16a) that if this basis is an eigenbasis of $\rho_{2}$, then and only then, the general expansion (20a) takes the special form of the biorthogonal Schmidt expansion (18).

Finally, one has

$$
\rho_{1}=A_{a}^{\dagger} A_{a}, \quad \rho_{2}=A_{a} A_{a}^{\dagger}
$$

The correlation operator $U_{a}$ establishes a striking mathematical symmetry and close connection between the two closed ranges $\overline{\mathcal{R}}\left(\rho_{s}\right), s=1,2$, for any bipartite state vector $|\Phi\rangle_{12}$. The pair of entities $\rho_{1}, U_{a}$, which is equivalent to $|\Phi\rangle_{12}$ (cf (9a) and (3)), is called the correlated subsystem picture of the given bipartite state vector. (Note that when one takes a state vector $|\Phi\rangle_{12}$ instead of a state $|\Phi\rangle_{12}\left\langle\left.\Phi\right|_{12}\right.$, the former is informationally richer by the choice of a fixed phase factor $e^{i \lambda}, \lambda \in \mathbf{R}$, which is arbitrary in the latter. This choice is carried by $A_{a}$ or $U_{a}$. Thus, $U_{a}$ and $e^{i \lambda} U_{a}$ with the same $\rho_{1}$, correspond to $|\Phi\rangle_{12}$ and $e^{i \lambda}|\Phi\rangle_{12}$ respectively.) 
We have summed up in this Subsection the mathematical part of the antilinear representation of $|\Phi\rangle_{12}$, and of the correlated subsystem picture. The basic physical meaning of these was studied in previous articles. ${ }^{2,8} \mathrm{~A}$ summary is given in the next subsection.

\section{B. The physical part - detectably-complete state- compatible observables}

Returning to the general expansion (13a), it can be completed by

$$
\begin{gathered}
\rho_{2}=\sum_{j}\left(A_{a}|j\rangle_{1}\right)_{2}\left(\left\langle\left. j\right|_{1} A_{a}^{\dagger}\right)_{2}=\sum_{j} p_{j}\left|\phi_{j}\right\rangle_{2}\left\langle\left.\phi_{j}\right|_{2},\right.\right. \\
\forall j: \quad p_{j} \equiv \|\left(A_{a}|j\rangle_{1}\right)_{2} \|^{2} \\
\forall j, \quad p_{j}>0: \quad\left|\phi_{j}\right\rangle_{2} \equiv p_{j}^{-1 / 2}\left(A_{a}|j\rangle_{1}\right)_{2} .
\end{gathered}
$$

Here $p_{j}$ is the probability that the event $\left(|j\rangle_{1}\left\langle\left. j\right|_{1} \otimes 1\right)\right.$ occurs in nearbysubsystem measurement in $|\Phi\rangle_{12}$, and $\left|\phi_{j}\right\rangle_{2}$ is the state of the remote subsystem thus obtained, i. e., it is the result of so-called remote preparation. From the non-selective (or entire-ensemble) point of view, the physical meaning of $(13 \mathrm{a}-\mathrm{d})$ consists in the fact that these relations express a remote complete state decomposition in the antilinear representation. (In remote incomplete state decomposition also mixed states of the remote subsystem are obtained. Such a remote decomposition is given rise to by incomplete nearby-subsystem measurement, i. e., by measurement of an observable with degenerate eigenvalues.)

A detectably complete (see below) nearby subsystem observable $A_{1}$ that is compatible with the nearby subsystem state, i. e., that satisfies $\left[A_{1}, \rho_{1}\right]=0$, shortly, a state-compatible observable, has, on account of this relation, as it is well known, a common eigenbasis with $\rho_{1}$. Let its subbasis spanning $\overline{\mathcal{R}}\left(\rho_{1}\right)$ be $\left\{\left|r_{i}\right\rangle_{1}: \forall i\right\} \quad(\operatorname{cf}(7)$ and (8a)). Then the relevant 
partial spectral form of $A_{1}$ is

$$
A_{1}=\sum_{i} a_{i}\left|r_{i}\right\rangle_{1}\left\langle\left. r_{i}\right|_{1}+Q_{1}^{\perp} A_{1}, \quad i \neq i^{\prime} \Rightarrow a_{i} \neq a_{i^{\prime}}\right.
$$

where $Q_{1}=\sum_{i}\left|r_{i}\right\rangle_{1}\left\langle\left. r_{i}\right|_{1}\right.$ is the range projector of $\rho_{1}, \quad Q_{1}^{\perp}$ is the orthocomplemnentary projector, and the sum in (21a) is the detectable part (in $\overline{\mathcal{R}}\left(\rho_{1}\right)$ ) of $A_{1}$.

By detectably complete is meant the requirement in (21a), i. e., completeness of the reducee $\widetilde{A_{1}}=\sum_{i} a_{i}\left|r_{i}\right\rangle r_{i} \mid$ of $A_{1}$ in $\overline{\mathcal{R}}\left(\rho_{1}\right)$. (For the use of tilde cf $\tilde{\rho}_{2}$ in the passage beneath $(9 \mathrm{a}, \mathrm{b})$.)

When $A_{1}$ is measured (in an ideal way, e. g.), it gives rise to the actual state decomposition (empirically ensemble decomposition):

$$
\rho_{2}=\sum_{i} r_{i}\left|r_{i}\right\rangle_{2}\left\langle\left. r_{i}\right|_{2}\right.
$$

(special case of $(13 \mathrm{a}-\mathrm{d}))$. Since the state vectors $\left\{\left|r_{i}\right\rangle_{2}: \forall i\right\}$ are orthogonal (cf $(7)$ and $(8 b)),(21 b)$ amounts to the same as if a detectably complete remote-subsystem observable (Hermitian operator)

$$
A_{2}=\sum_{i} a_{i}^{\prime}\left|r_{i}\right\rangle_{2}\left\langle\left. r_{i}\right|_{2}+Q_{2}^{\perp} A_{2}, \quad i \neq i^{\prime} \Rightarrow a_{i}^{\prime} \neq a_{i^{\prime}}^{\prime}\right.
$$

had been measured in an ideal way. Here $Q_{2}=\sum_{i}\left|r_{i}\right\rangle_{2}\left\langle\left. r_{i}\right|_{2}\right.$ is the range projector of $\rho_{2}$.

The pairs of observables $\left(A_{1}, A_{2}\right)$ are called (physical) twin observables, the indirect measurement of $A_{2}$ by measuring $A_{1}$ directly is called remote measurement, and the twin observables satisfy the symmetric relations

$$
\begin{gathered}
{\left[A_{1}, \rho_{1}\right]=0, \quad\left[A_{2}, \rho_{2}\right]=0} \\
A_{2}=\sum_{i} a_{i}^{\prime}\left(U_{a}\left(\left|r_{i}\right\rangle_{1}\left\langle\left. r_{i}\right|_{1}\right) U_{a}^{-1}\right)_{2} Q_{2}+Q_{2}^{\perp} A_{2},\right.
\end{gathered}
$$




$$
A_{1}=\sum_{i} a_{i}\left(U_{a}^{-1}\left(\left|r_{i}\right\rangle_{2}\left\langle\left. r_{i}\right|_{2}\right) U_{a}\right)_{1} Q_{1}+Q_{1}^{\perp} A_{1}\right.
$$

In (22c) it is assumed that $A_{1}=\sum a_{i}\left|r_{i}\right\rangle_{1}\left\langle\left. r_{i}\right|_{1}+Q_{1}^{\perp} A_{1}\right.$ is given, and $A_{2}$ is determined by it (at least as far as the eigenvectors of the detectable part of $A_{2}$ are concerned). In (22d) the symmetrical assumption is made. One should note that the undetectable parts $Q_{1}^{\perp} A_{1}$ and $Q_{2}^{\perp} A_{2}$ are completely arbitrary (and so are the distinct detectable eigenvalues of the twin operator).

In Ref-s 2 and 8, it was assumed that the detectable spectra coincide:

$$
\forall i: \quad a_{i}^{\prime}=a_{i}
$$

Then

$$
A_{2}=\left(U_{a} A_{1} U_{a}^{-1}\right)_{2} Q_{2}+Q_{2}^{\perp} A_{2}
$$

and

$$
A_{1}=\left(U_{a}^{-1} A_{2} U_{a}\right)_{1} Q_{1}+Q_{1}^{\perp} A_{1}
$$

are valid. In later work, ${ }^{10}$ twin observables with the stronger requirement (23a) were called algebraic twin observables. Relaxation of the stronger requirement led to the wider and more useful class of physical twin observables.

If one exchanges the roles of subsystems 1 and 2 , one can measure $A_{1}$ remotely by a direct measurement of $A_{2}$.

Thus, part of the physical meaning of the correlation operator $U_{a}$ inherent in $|\Phi\rangle_{12}$ is in the following: When a detectably complete nearbysubsystem observable $A_{1}$ (cf (21a)) that is compatible with the nearbysubsystem state is measured in an ideal way in selective measurement and $a_{i}$ is obtained as a result, then the nearby subsystem is found in the state $\left|r_{i}\right\rangle_{1}$, and the remote subsystem is in the state $\left|r_{i}\right\rangle_{2} \equiv\left(U_{a}\left|r_{i}\right\rangle_{1}\right)_{2}$ (conditional state). It is obvious from (18) and (19a), that also the symmetrical argument is valid. The correlation operator (and its inverse) give the 
corresponding conditional states when selective ideal measurement of statecompatible subsystem observables is performed.

The remote measurement of a twin observable $A_{2}$, selective or nonselective, is one and the same in every kind of measurement of $A_{1}$ : in ideal measurement and in second-kind (synonym: non-repeatable) measurement (cf Subsection 6(B) in Ref. 2).

\section{LINEARLY-INDEPENDENT COMPLETE DECOM- POSITIONS OF DENSITY OPERATORS}

Definition 1: A finite or countably infinite set of vectors $\left\{\left|\phi_{i}\right\rangle: \forall i\right\}$ is said to be linearly independent if

$$
\forall i: \quad\left|\phi_{i}\right\rangle \notin \overline{\operatorname{span}}\left\{\left|\phi_{i^{\prime}}\right\rangle: \forall i^{\prime}, i^{\prime} \neq i\right\},
$$

where by " $\overline{\text { span }} "$ is meant the algebraic and topological span, i. e., the set of all linear combinations together with all their limiting points. (It is a subspace.)

One can define linear independence of a finite sequence $\left\{\left|\phi_{i}\right\rangle: i=\right.$ $1,2, \ldots, d<\infty\}$ of vectors by the weaker requirement:

$$
\forall k, \quad k \geq 2:\left|\phi_{k}\right\rangle \notin \operatorname{span}\left\{\left|\phi_{1}\right\rangle, \ldots,\left|\phi_{(k-1)}\right\rangle\right\} .
$$

Proof is given in Appendix A. (Note that finite-dimensional linear manifolds are subspaces, i. e., $\quad$ span $=\overline{\text { span }}$ in this case.)

Definition 2: If $\left\{\left|\phi_{i}\right\rangle: i=1,2, \ldots, d \leq \infty\right\}$ is a linearly-independent finite or infinite sequence, $\rho$ a density operator with a $d$-dimensional closed 
range, and if one can write

$$
\rho=\sum_{i} p_{i}\left|\phi_{i}\right\rangle\left\langle\phi_{i}\right|
$$

where $\forall i: \quad p_{i}>0, \quad \sum_{i} p_{i}=1$, then one speaks of a linearly-independent complete decomposition of the density operator. (It is called "irreducible decomposition" in Ref-s 11 and 4.)

We call "complete" those decompositions of a density operator that cannot be continued by further decomposing any term. These are the pure-state decompositions quantum mechanically. In a followup to this article we turn to "incomplete" decompositions, i. e., to mixed-or-pure state decompositions quantum mechanically.

For $(26 a)$ the relation

$$
\overline{\mathcal{R}}(\rho)=\overline{\operatorname{span}}\left\{\left|\phi_{i}\right\rangle: \forall i\right\}
$$

is valid (cf Proposition 1 in Ref. 11).

Corollary 1: Obviously, orthonormal sets are special cases of linearly independent ones. The latter possess some important properties of the former. One of them is the following. If $\left\{\left|\phi_{i}\right\rangle: i=1,2, \ldots, d \leq \infty\right\}$ is a linearly-independent sequence, $k$ is an integer not larger than $d$, and $\left\{\left|\phi_{1}\right\rangle, \ldots,\left|\phi_{k}\right\rangle\right\}$ is a subset of arbitrary elements in arbitrary order, then it spans a $k$-dimensional subspace $\mathcal{S}_{k}$, and each vector in it can be uniquely expanded in the set. This is why the latter is called a linearly-independent basis in $\mathcal{S}_{k}$. (See also Corollary 3 below.)

Proof is given in Appendix B. 
Now we sum up those results on density-operator decomposition from Ref. 4 the application of which forms the basis of this work. They are further elaborated in this section with a view to help applications in quantum-mechanical studies. (No heed is paid to the extent to which the elaborations are possibly new with respect to the mathematical literature, cf e. g. Ref. 12.)

Lemma: A) Let $\rho$ be an arbitrary given density operator, and let $d$ be the dimension of its (finite or infinite dimensional) closed range. Then all linearly-independent sequences $\left\{\left|\phi_{i}\right\rangle: i=1,2, \ldots, d\right\}$ that determine a complete decomposition

$$
\rho=\sum_{i} p_{i}\left|\phi_{i}\right\rangle\left\langle\phi_{i}\right|
$$

of $\rho$ stand in a one-to-one relation with the set of all bases in $\overline{\mathcal{R}}(\rho)$ each vector of which is within $\mathcal{R}\left(\rho^{1 / 2}\right)$ :

$$
\left\{\left|e_{i}\right\rangle: i=1,2, \ldots, d \leq \infty\right\} \subset \mathcal{R}\left(\rho^{1 / 2}\right),
$$

where $d$ is the dimension of $\overline{\mathcal{R}}(\rho)$.

B) The bijection from the set of all bases (28) to all linearly-independent sequences that give decompositions (27) - we call it the Cassinelli-VitoLevrero (CVL) bijection - reads as follows:

$p_{i}=\left\langle e_{i}|\rho| e_{i}\right\rangle=\| \rho^{1 / 2}\left|e_{i}\right\rangle \|^{2}>0, \quad\left|\phi_{i}\right\rangle=p_{i}^{-1 / 2} \rho^{1 / 2}\left|e_{i}\right\rangle, \quad i=1,2, \ldots, d \leq \infty$. $(29 a, b)$

The inverse CVL bijection is

$$
\left|e_{i}\right\rangle=p_{i}^{1 / 2} \tilde{\rho}^{-1 / 2}\left|\phi_{i}\right\rangle, \quad i=1,2, \ldots, d \leq \infty,
$$

where the tilde denotes the reducee in $\overline{\mathcal{R}}(\rho)$. 
C) Finally, a state vector $|\phi\rangle$ can appear in a linearly-independent complete decomposition of $\rho$ if and only if

$$
|\phi\rangle \in \mathcal{R}(\rho), \quad i=1,2, \ldots, d \leq \infty .
$$

For proof see Theorem 1, Proposition 1, and Remark 8 in Ref. 4.

In connection with the Lemma, one should keep in mind the well-known (and easily proved) relations

$$
\mathcal{R}(\rho) \subseteq \mathcal{R}\left(\rho^{1 / 2}\right) \subseteq \overline{\mathcal{R}}\left(\rho^{1 / 2}\right)=\overline{\mathcal{R}}(\rho) .
$$

In case of finite-dimensional range, one has equality all over. Contrarily, in case of infinite-dimensional range, both subset relations in (32) are proper.

Corollary 2: The CVL bijection is non-trivial if and only if the basis (28) is not an eigen-sub-basis of $\rho$ (otherwise, it is the identity map).

Corollary 3: Another property of linearly-independent sequences parallelling that of orthonormal ones is the following. If (27) is a linearlyindependent complete decomposition of a density operator, then each element $|\chi\rangle$ from the range $\mathcal{R}\left(\rho^{1 / 2}\right)$ can be uniquely expanded in the sequence $\left\{\left|\phi_{i}\right\rangle: i=1,2, \ldots, d \leq \infty\right\}$ :

$$
|\chi\rangle=\sum_{i} \alpha_{i}\left|\phi_{i}\right\rangle
$$

(cf (32)). Further, utilizing the scalar product, one has the following compact formula for the expansion coefficients:

$$
\alpha_{i}=p_{i}\left[\left(\left\langle\phi_{i}\right| \tilde{\rho}^{-1}\right)|\chi\rangle\right], \quad i=1,2, \ldots, d \leq \infty
$$


(cf Lemma C)). In this sense, the sequence at issue is a linearly-independent basis in $\mathcal{R}\left(\rho^{1 / 2}\right)$.

Note that the uniqueness of expansion (33a) allows an arbitrary (hence, if desired, a suitable) choice of the probability distribution $\left\{p_{i}: i=1,2, \ldots, d \leq\right.$ $\left.\infty ; p_{i}>0 ; \sum_{i=1}^{d} p_{i}=1\right\}$, and the definition of $\rho$ via (27). (But care must be taken that $\mathcal{R}\left(\rho^{1 / 2}\right)$ contain $|\chi\rangle$. ) Note, further, that all $d$ probabilities $p_{i}$ must be positive. Otherwise, $|\chi\rangle$ would not be expanded in the linearly-independent basis $\left\{\left|\phi_{i}\right\rangle: i=1,2, \ldots, d \leq \infty\right\}$.

Corollary 3 is proved in Appendix C.

Corollary 4: If (27) is a linearly-independent complete decomposition of a given density operator, then the weight $p_{i}$ can also be expressed in the following two ways:

$$
p_{i}=\left(\left\langle\phi_{i}\left|\tilde{\rho}^{-1}\right| \phi_{i}\right\rangle\right)^{-1}, \quad i=1,2, \ldots, d \leq \infty,
$$

and

$$
p_{i}=1 /\left(\sum_{k}\left(\left|\left\langle k|| \phi_{i}\right\rangle\right|^{2} r_{k}^{-1}\right)\right), \quad i=1,2, \ldots, d \leq \infty
$$

where

$$
\rho=\sum_{k} r_{k}|k\rangle\langle k|, \quad \forall k: \quad r_{k}>0
$$

is a complete spectral decomposition of $\rho$.

Further, one has

$\inf \left\{r_{k}:\left|\left\langle\phi_{i}|| k\right\rangle\right|^{2}>0\right\} \leq p_{i} \leq \max \left\{r_{k}:\left|\left\langle\phi_{i}|| k\right\rangle\right|^{2}>0\right\}, \quad i=1,2, \ldots, d \leq \infty$,

where the "infimum" can be raplaced by "minimum" if the range of $\rho$ is finite dimensional. 
Proof: Expression (34) is obtained by taking the square norm of both sides of (30). Expression (35a) follows from (34) when $\left|\phi_{i}\right\rangle$ is expanded in the eigen-sub-basis $\{|k\rangle: \forall k\}$ of $\rho$ (and eigenbasis of $\tilde{\rho}$.) Finally, inequalities (36) are an immediate consequence of (35a).

Remark 1: When a density operator $\rho$ is given and a state vector satisfies $|\phi\rangle \in \mathcal{R}(\rho) \quad$ ( cf Lemma C)), then, in whatever linearly-independent complete decomposition of the former the latter appears, it has a unique weight $p$, which depends only on $\rho$ and $|\phi\rangle \quad$ (cf (34)).

Definition 3: We call the weight $p$ from Remark 1 the characteristic weight of $|\phi\rangle$ in $\rho$. If $|\phi\rangle \notin \mathcal{R}(\rho)$, then $p \equiv 0$.

Note that if $|\phi\rangle \in \mathcal{R}(\rho)$, then $p>0$ (cf (30a)). Note, further, that Remark 1 and Corollary 4 are a completion of Lemma C.

Remark 2: For a possible positive value of the characteristic weight $p$, there may be more than one corresponding state vector $|\phi\rangle$ in a linearlyindependent complete state decomposition (27) as seen from (29a), because more than one state vector $|f\rangle$ can give one and the same expectation value of $\rho$, and each can be the first $\left|e_{1}\right\rangle \equiv|f\rangle$ in a basis etc. (cf the Lemma).

Corollary 5: The characteristic weight $p$ of a given state vector $|\phi\rangle \in$ $\mathcal{R}(\rho)$ satisfies the inequality:

$$
p \leq\langle\phi|\rho| \phi\rangle .
$$

One has $p=\langle\phi|\rho| \phi\rangle$ if and only if $|\phi\rangle$ is an eigenvector of $\rho$, and 
then $p$ equals the corresponding eigenvalue of the density operator.

Proof: The inequality (37) follows from (27) when one puts $\left|\phi_{1}\right\rangle \equiv|\phi\rangle$ in (27), and one obtains

$$
\rho=p|\phi\rangle\left\langle\phi\left|+\sum_{i=2} p_{i}\right| \phi_{i}\right\rangle\left\langle\phi_{i}\right|
$$

one applies $|\phi\rangle\langle\phi|$ to both sides, and one takes the trace (keeping in mind, of course, that $\operatorname{tr}(|\phi\rangle\langle\phi| \rho)=\langle\phi|\rho| \phi\rangle)$ :

$$
\langle\phi|\rho| \phi\rangle=p+\sum_{i=2} p_{i}\left|\left\langle\phi|| \phi_{i}\right\rangle\right|^{2} \geq p
$$

One can see from (34) that if $|\phi\rangle$ is an eigenvector of $\rho$ corresponding to the eigenvalue $r$, then $p=r$, and also $\langle\phi|\rho| \phi\rangle=r=p$. Conversely, if $p=\langle\phi|\rho| \phi\rangle$, then one can see from (38b) that all vectors $\left\{\left|\phi_{i}\right\rangle: i=2,3, \ldots\right\}$ must be orthogonal to $|\phi\rangle$. Hence, applying (38a) to $|\phi\rangle$, it is seen that the latter is an eigenvector of $\rho$ corresponding to the eigenvalue $r=p$.

\section{REMOTE LINEARLY-INDEPENDENT COMPLETE STATE DECOMPOSITION}

Let $|\Phi\rangle_{12}$ be an arbitrary bipartite state vector. Owing to the Cassinelli et al. theory, summed up in the Lemma, we can now easily sort out what kind of local, i. e., subsystem measurement gives rise to a linearly-independent complete decomposition of the opposite-subsystem state.

Definition 4: Since subsystem measurement, by definition, excludes any interaction between the measuring instrument and the remote subsystem, we call any influence of the former on the latter, which is due exclusively to the 
quantum correlations inherent in the bipartite state, remote influence.

Definition 5: We call a nearby subsystem observable $A_{1}$ relevant (for remote linearly-independent complete state decomposition) if the following three conditions are satisfied:

(i)

$$
\left[A_{1}, Q_{1}\right]=0
$$

where $Q_{1}$ is the range projector of $\rho_{1} \equiv \operatorname{tr}_{2}\left(|\Phi\rangle_{12}\left\langle\left.\Phi\right|_{12}\right)\right.$. If (39) is satisfied, then $A_{1}$ will be said to be range compatible.

(ii) $\tilde{A}_{1}$, the reducee of $A_{1}$ in $\overline{\mathcal{R}}\left(\rho_{1}\right)$ if (39) is satisfied, has a purely discrete and non-degenerate spectrum.

(iii) The eigenbasis $\left\{\left|e_{i}\right\rangle_{1}: \forall i\right\}$ of $\tilde{A}_{1}$ in $\overline{\mathcal{R}}\left(\rho_{1}\right) \quad$ (which is uniquely determined by $\tilde{A}_{1}$ up to arbitrary phase factors and ordering) is within $\mathcal{R}\left(\rho_{1}^{1 / 2}\right)$. (This requirement is always satisfied if the dimension $d$ of $\rho_{1}$ is finite).

Further, we call a basis $\left\{\left|e_{i}\right\rangle_{1}: \forall i\right\}$ in $\overline{\mathcal{R}}\left(\rho_{1}\right)$ that is entirely within $\mathcal{R}\left(\rho_{1}^{1 / 2}\right)$ relevant. Finally, we call a class of observables $A_{1}$ relevant if it consists of relevant observables that have one and the same relevant basis $\left\{\left|e_{i}\right\rangle_{1}: \forall i\right\} \quad$ (up to phase factors and ordering) as their eigen-sub-basis in $\overline{\mathcal{R}}\left(\rho_{1}\right)$.

Evidently, the set of all relevant classes of observables $A_{1}$ is in a simple one-to-one relation with the set of all relevant bases in $\mathcal{R}\left(\rho_{1}^{1 / 2}\right)$.

If $A_{1}$ is state-compatible, i. e., $\left[A_{1}, \rho_{1}\right]=0$, then $A_{1}$ commutes also with every eigenprojector of $\rho_{1}$, and hence (39) is satisfied. Namely, $Q_{1}$ is the sum of the eigenprojectors corresponding to positive eigenvalues. (If $\mathcal{R}\left(\rho_{1}\right)$ is infinite dimensional, we can assume that $A_{1}$ is bounded, 
or, equivalently, continuous, or equivalently, that its spectrum is within a finite interval. We can do this because the spectrum of $A_{1}$ is arbitrary within the relevant class of observables, i. e., it is irrelevant for remote state decomposition.)

In this case the reducee $\tilde{A}_{1}$ has necessarily a purely discrete spectrum (because it reduces in every eigen-subspace of $\rho_{1}$, and these are necessarily finite dimensional due to the fact that the corresponding eigenvalues add up to 1). Thus, in this case, requirement (i) is necessarily fulfilled, and (ii) reads that $A_{1}$ is a detectably complete observable, i. e., that $\tilde{A}_{1}$ is complete. Requirement (iii) is necessarily satisfied because $\left[A_{1}, \rho_{1}\right]=0$ entails a common eigenbasis of $\tilde{A}_{1}$ and $\tilde{\rho}_{1}$. Further, the corresponding spectral form of $\tilde{\rho}_{1}$ is simultaneously a complete decomposition of it. Hence, according to the known result of Hadjisavvas, ${ }^{11}$ each of the eigenvectors necessarily belongs to $\mathcal{R}\left(\rho_{1}^{1 / 2}\right)$.

Theorem 1: A) If a relevant observable $\left(A_{1} \otimes 1\right)$ is measured in the state $|\Phi\rangle_{12}$, it gives rise to a remote linearly-independent complete decomposition of the state $\rho_{2} \equiv \operatorname{tr}_{1}\left(|\Phi\rangle_{12}\left\langle\left.\Phi\right|_{12}\right)\right.$ :

$$
\rho_{2}=\sum_{i} p_{i}\left|\phi_{i}\right\rangle_{2}\left\langle\left.\phi_{i}\right|_{2}\right.
$$

Conversely, each mathematically possible linearly-independent complete decomposition of $\rho_{2}$ can be obtained in this way.

B) The mathematical way how $A_{1}$ determines (40) can be understood as a bijection of the set of all classes of detectably equivalent observables $A_{1}$, or, equivalently, of all relevant bases $\left\{\left|e_{i}\right\rangle_{1}: \forall i\right\}$, onto the set of all linearly-independent complete decompositions (40) $(\mathrm{A} \searrow \mathrm{D}$ on Diagram 1 
below) that reads:

$$
\begin{gathered}
\forall i: \quad p_{i}=\left\langle\Phi | _ { 1 2 } \left(\left|e_{i}\right\rangle_{1}\left\langle\left. e_{i}\right|_{1} \otimes 1\right)|\Phi\rangle_{12}>0,\right.\right. \\
\forall i: \quad\left|\phi_{i}\right\rangle_{2}=p_{i}^{-1 / 2} \rho_{2}^{1 / 2}\left(U_{a}\left|e_{i}\right\rangle_{1}\right)_{2}
\end{gathered}
$$

where $\left|e_{i}\right\rangle_{1}$ are the eigenbasis vectors of $\tilde{A}_{1}=\sum_{i^{\prime}} a_{i^{\prime}}\left|e_{i^{\prime}}\right\rangle_{1}\left\langle\left. e_{i^{\prime}}\right|_{1}\right.$. Further, $U_{a}$ is the antiunitary correlation operator determined by $|\Phi\rangle_{12}$ (cf (9a,b) and the passage beneath it, as well as the passage beneath (12c)).

C) The inverse bijection ( $A \nwarrow D$ on the diagram) has the form:

$$
\forall i: \quad\left|e_{i}\right\rangle_{1}=\left(U_{a}^{-1}\left(p_{i}^{1 / 2} \tilde{\rho}_{2}^{-1 / 2}\left|\phi_{i}\right\rangle_{2}\right)\right)_{1}
$$

All claims symmetric to those in A)-C) are also valid:

D) If $\left(1 \otimes A_{2}\right)=\sum_{j} a_{j}^{\prime}\left(1 \otimes\left|f_{j}\right\rangle_{2}\left\langle\left. f_{j}\right|_{2}\right)+\left(1 \otimes Q_{2}^{\perp} A_{2}\right) \quad\right.$ is the relevant partial spectral form of an arbitrary relevant observable $A_{2} \quad\left(Q_{2}\right.$ being the range projector of $\rho_{2} \equiv \operatorname{tr}_{1}\left(|\Phi\rangle_{12}\left\langle\left.\Phi\right|_{12}\right)\right.$ ), its (non-selective) measurement causes a remote linearly-independent complete state decomposition

$$
\rho_{1} \equiv \operatorname{tr}_{2}\left(|\Phi\rangle_{12}\left\langle\left.\Phi\right|_{12}\right)=\sum_{j} q_{j}\left|\chi_{j}\right\rangle_{1}\left\langle\left.\chi_{j}\right|_{1}, \quad \forall j: \quad q_{j}>0, \quad \sum_{j} q_{j}=1 .\right.\right.
$$

Each linearly-independent complete decomposition of $\rho_{1}$ can be obtained in this way.

E) The bijection $\left(\mathrm{C}_{\swarrow} \mathrm{B}\right.$ on the diagram) taking the set of all relevant classes of second-subsystem observables onto that of all linearly-independent complete first-subsystem state decompositions reads:

$$
\forall j: \quad q_{j} \equiv\left\langle\Phi | _ { 1 2 } \left( 1 \otimes\left|f_{j}\right\rangle_{2}\left\langle\left. f_{j}\right|_{2}\right)|\Phi\rangle_{12}>0\right.\right.
$$




$$
\forall j: \quad\left|\chi_{j}\right\rangle_{1} \equiv q_{j}^{-1 / 2} \rho_{1}^{1 / 2}\left(U_{a}^{-1}\left|f_{j}\right\rangle_{2}\right)_{1}
$$

F) The inverse bijection $(\mathrm{C} \nearrow \mathrm{B})$ is

$$
\forall j: \quad\left|f_{j}\right\rangle_{2} \equiv\left(U_{a}\left(q_{j}^{1 / 2} \tilde{\rho}_{1}^{-1 / 2}\left|\chi_{j}\right\rangle_{1}\right)\right)_{2} .
$$

G) A bijection mapping all relevant classes of observables $\left(A_{1} \otimes 1\right)$ onto that of all relevant classes of observables $\left(1 \otimes A_{2}\right) \quad(\mathrm{A} \longrightarrow \mathrm{B}$ on the diagram $)$ is

$$
\forall i: \quad\left|f_{i}\right\rangle_{2} \equiv\left(U_{a}\left|e_{i}\right\rangle_{1}\right)_{2}
$$

The inverse bijection ( $\mathrm{A} \longleftarrow \mathrm{B}$ on the diagram) is

$$
\forall j: \quad\left|e_{j}\right\rangle_{1} \equiv\left(U_{a}^{-1}\left|f_{j}\right\rangle_{2}\right)_{1}
$$

H) The product bijection $\left(\mathrm{C}_{\swarrow} \mathrm{B}\right) \circ(\mathrm{A} \longrightarrow \mathrm{B})$ ("o" meaning "after") is the corresponding CVL bijection $(A \downarrow C)$; and symmetrically, the product bijection $(\mathrm{A} \searrow \mathrm{D}) \circ(\mathrm{A} \longleftarrow \mathrm{B})$ is the corresponding CVL bijection $(B \downarrow D)$.

I) A bijection taking all linearly-independent complete decomposition of $\rho_{1}$ onto those of $\rho_{2} \quad(\mathrm{C} \longrightarrow \mathrm{D})$ is

$$
\left[U_{a}\left(\rho_{1}=\sum_{i} q_{i}\left|\chi_{i}\right\rangle_{1}\left\langle\left.\chi_{i}\right|_{1}\right) U_{a}^{-1}\right]_{2} Q_{2}\right.
$$

giving, due to (10),

$$
\rho_{2}=\sum_{j} p_{j}\left|\phi_{j}\right\rangle_{2}\left\langle\left.\phi_{j}\right|_{2},\right.
$$


where

$$
\begin{gathered}
\forall j: \quad p_{j} \equiv q_{j}, \\
\forall j: \quad\left|\phi_{j}\right\rangle_{2} \equiv\left(U_{a}\left|\chi_{j}\right\rangle_{1}\right)_{2} .
\end{gathered}
$$

The inverse bijection ( $\mathrm{C} \longleftarrow \mathrm{D}$ on the diagram) is symmetric to this (under the exchange of the two subsystems) mutatis mutandis.

J) The square Diagram 1 summing up the preceding items of Theorem 1 is commutative, i. e., any two successive bijections multiply into the corresponding bijection on the Diagram.

\section{Commutative Square Diagram 1. \\ A Mathematical Framework for Remote Linearly-Independent Complete State Decompositions}
$\mathbf{A} \equiv\left\{\right.$ all relevant classes of $\left.A_{1}\right\}$
$\mathbf{B} \equiv\left\{\right.$ all relevant classes of $\left.A_{2}\right\}$

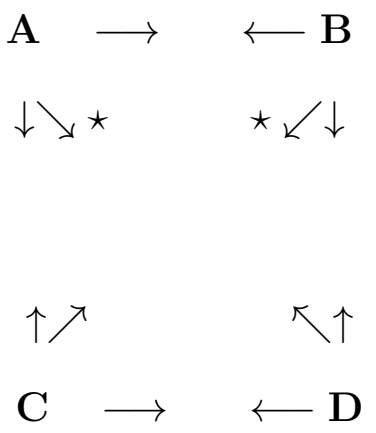

$\mathbf{C} \equiv\left\{\right.$ all linearly-independent complete decompositions of $\left.\rho_{1}\right\}$
$\mathbf{D} \equiv\left\{\right.$ all linearly-independent complete decompositions of $\left.\rho_{2}\right\}$ 
Caption. Each arrow goes from one of the sets (A,B,C,D) towards another. It stands for the corresponding bijection. Oppositely oriented arrows denote mutually inverse bijections. The diagram is commutative, i. e., the successive bijections combine into the displayed corresponding one. For instance, taking the bijection $\mathbf{B} \downarrow \mathbf{D}$ after the bijection $\mathbf{A} \rightarrow \mathbf{B}$ gives the bijection $\mathbf{A} \searrow \mathbf{D}$. The bijections are given in detail in Theorem 1 .

The imaginary vertical line cutting the square into two equally wide halves makes these completely symmetric (due to the symmetry between the two subsystems in $|\Phi\rangle_{12}$ ).

$\star$ The downward diagonal bijections $\mathbf{A} \searrow \mathbf{D}$ and $\mathbf{C} \swarrow \mathbf{B}$ have the physical meaning of remote linearly-independent state decompositions.

\section{Proof of Theorem 1.}

B) To prove claim B, we take resort to the relations (13a-d), which express the general remote complete state decomposition. Relation (13c) and (3) imply in our case

$$
\begin{gathered}
\forall i: \quad 0<p_{i}=\|\left(A_{a}\left|e_{i}\right\rangle_{1}\right)_{2}\left\|^{2}=\right\|\left\langle\left. e_{i}\right|_{1} \mid \Phi\right\rangle_{12} \|^{2}= \\
\left(\left(\left\langle\left.\Phi\right|_{12} \mid e_{i}\right\rangle_{1}\right)_{2},\left(\left\langle\left. e_{i}\right|_{1} \mid \Phi\right\rangle_{12}\right)_{2}\right)=\left\langle\Phi | _ { 1 2 } \left(\left|e_{i}\right\rangle_{1}\left\langle\left. e_{i}\right|_{1} \otimes 1\right)|\Phi\rangle_{12} .\right.\right.
\end{gathered}
$$

Further, (13d) and (9b) give $\forall i:\left|\phi_{i}\right\rangle_{2}=p_{i}^{-1 / 2} \rho_{2}^{1 / 2}\left(U_{a}\left|e_{i}\right\rangle_{1}\right)_{2}$.

C) Claim $\mathrm{C}$ obviously follows from $\mathrm{B}$ in view of the facts that both $U_{a}$ and $\quad \tilde{\rho}_{2}^{1 / 2}$ are non-singular on $\overline{\mathcal{R}}\left(\rho_{1}\right)$ and in $\overline{\mathcal{R}}\left(\rho_{2}\right)$ respectively.

A) The proof of claim $\mathrm{A}$ is a consequence of part of the commutativity of the square Diagram, viz., of the fact that $(A \searrow D)=(B \downarrow D) \circ(A \longrightarrow B)$. To see this, one should keep in mind that $\rho_{2}=\left(U_{a} \rho_{1} U_{a}^{-1}\right)_{2} Q_{2} \quad(\operatorname{cf}(10))$ implies $U_{a} \mathcal{R}\left(\rho_{1}^{1 / 2}\right)=\mathcal{R}\left(\rho_{2}^{1 / 2}\right)$ because the definition $\rho_{1}^{1 / 2} \rho_{1}^{1 / 2}=\rho_{1}$ of the square root, (10) and the well known uniqueness of the square root lead to $\left(U_{a} \rho_{1}^{1 / 2} U_{a}^{-1}\right)_{2} Q_{2}\left(U_{a} \rho_{1}^{1 / 2} U_{a}^{-1}\right)_{2} Q_{2}=\rho_{2}$, and finally to $\left(U_{a} \rho_{1}^{1 / 2} U_{a}^{-1}\right)_{2} Q_{2}=$ $\rho_{2}^{1 / 2}$. Therefore, $\left\{\left|e_{i}\right\rangle_{1}: \forall i\right\} \subset \mathcal{R}\left(\rho_{1}^{1 / 2}\right)$ implies $\left\{\left(U_{a}\left|e_{i}\right\rangle_{1}\right)_{2}: \forall i\right\} \subset$ $\mathcal{R}\left(\rho_{2}^{1 / 2}\right)$.

Further, let us rewrite (41a) as

$$
\forall i: \quad p_{i}=\left\langle\left. e_{i}\right|_{1} \rho_{1} \mid e_{i}\right\rangle_{1}=\left(\left\langle\left. e_{i}\right|_{1} U_{a}^{\dagger}\right)_{2}\left(U_{a} \rho_{1} U_{a}^{-1}\right)_{2}\left(U_{a}\left|e_{i}\right\rangle_{1}\right)_{2}=\right.
$$




$$
\left(\left\langle\left. e_{i}\right|_{1} U_{a}^{\dagger}\right) \rho_{2}\left(U_{a}\left|e_{i}\right\rangle_{1}\right)_{2}\right.
$$

(Complex conjugation due to applying an antilinear operator to the left is omitted because the scalar product is a positive number.)

Comparing the last relation with (29a), and (41b) with (29b), we see that the claimed product of maps holds true. Since the factors in the product are bijections, so is the product itself (and its inverse is the reverse product of the inverses).

Finally, on account of the fact that the CVL bijection $(B \downarrow D)$ maps onto the set of all linearly-independent complete state decompositions (in $\left.\mathcal{R}\left(\rho_{2}\right)\right)$, the same is valid for the remote state decompositions $(A \searrow D)$ as claimed.

The symmetric claims D, E, and F can be proved symmetrically. Claim $\mathrm{G}$ is obviously valid.

H) Claim $\mathrm{H}$ is an immediate consequence of the products $(C \swarrow B)=$ $(A \downarrow C) \circ(A \longleftarrow B)$, which is the symmetric relation of $(A \searrow D)=(B \downarrow$ $D) \circ(A \longrightarrow B) \quad$ (see proof of claim $\mathrm{C}$ ).

Claim I is obviously valid. The final claim J easily follows from the multiplications proved for claim C.

Remark 3: In the special case when a pair of range-compatible observables $A_{s} s=1,2$, are state-compatible, then the CVL bijections $(A \downarrow C)$ and $(B \downarrow D)$ become mathematically most simple, and are endowed with physical meaning of actual orthogonal decomposition of the states $\rho_{s}, s=1,2$, due to ideal measurement. If the range-compatible observables are stateincompatible, then the CVL bijections are formal.

The remote linearly-independent complete decomposition (40), caused by 
the direct subsystem measurement of $\left(A_{1} \otimes 1\right)$, has the physical meaning of actual decomposition of $\rho_{2}$. This is so because, when the measurement interaction is over, the tripartite pure state vector has undergone the change

$$
\begin{gathered}
|0\rangle_{M A}|\Phi\rangle_{12}=|0\rangle_{M A}\left[\sum_{i}\left|e_{i}\right\rangle_{1}\left[\rho_{2}^{1 / 2}\left(U_{a}\left|e_{i}\right\rangle_{1}\right)_{2}\right]\right] \rightarrow \\
\sum_{i}|i\rangle_{M A}\left|e_{i}^{\prime}\right\rangle_{1}\left[\rho_{2}^{1 / 2}\left(U_{a}\left|e_{i}\right\rangle_{1}\right)_{2}\right]= \\
\sum_{i} p_{i}|i\rangle_{M A}\left|e_{i}^{\prime}\right\rangle_{1}\left|\phi_{i}\right\rangle_{2},
\end{gathered}
$$

where $|0\rangle_{M A}$ is the initial state vector of the measuring apparatus, and $\left\{|i\rangle_{M A}: \forall i\right\}$ is the orthonormal set of so-called "pointer positions": the state vectors in it display the results $\left\{a_{i}: \forall i\right\}$ in the measurement of $A_{1}$. The state vectors $\left|e_{i}^{\prime}\right\rangle_{1}$ equal the initial state vectors $\left|e_{i}\right\rangle_{1}$ if the measurement is a non-demolition (repeatable) one, and they differ otherwise. (Remember that we have complete measurement.) Anyway, according to (13a-d) (where now "1" is to be replaced by " $(M A+1)$ "), the final tripartite state gives rise to the remote state decomposition $\rho_{2}=\sum_{i} p_{i}\left|\phi_{i}\right\rangle_{2}\left\langle\left.\phi_{i}\right|_{2}\right.$. After reading the results on the measuring apparatus, i. e., after so-called objectivization, ${ }^{13}$ this decomposition becomes actual (in contrast to the infinitely-many mathematically possible so-called "potential" decompositions).

Returning to Theorem 1, and the square Diagram 1, we can say that the latter displays an extended physical meaning (with respect to that in Subsection 2.2) of the correlated subsystem picture.

Definition 6: Pairs of opposite subsystem observables $\left(A_{1}, A_{2}\right)$ satisfying $\left[A_{s}, Q_{s}\right]=0, s=1,2$, that are relevant (cf Definition 5) and can be written either as $A_{1}$ and $A_{2}=\sum_{i} a_{i}^{\prime}\left(U_{a}\left|e_{i}\right\rangle_{1}\right)_{2}\left(\left\langle\left. e_{i}\right|_{1} U_{a}^{\dagger}\right)_{2}+Q_{2}^{\perp} A_{2} \quad\right.$ or as $A_{1}=\sum_{i} a_{i}\left(U_{a}^{-1}\left|f_{i}\right\rangle_{2}\right)_{1}\left(\left\langle\left. f_{i}\right|_{2} U_{a}\right)_{1}+Q_{1}^{\perp} A_{1}\right.$ and $A_{2}$ (depending on 
the choice of the nearby and the remote subsystems), which amount to the same, can be called generalized twin observables. If $\left[A_{s}, \rho_{s}\right]=0, s=1,2$, are not valid, then one is dealing with extended twin observables.

Diagram 1 displays the physical meaning of the correlated subsystem picture that includes the extended twin observables (in addition to the special case of twin observables).

Corollary 6: The relevant classes of nearby-subsystem observables give an alternative classification of all linearly-independent complete decompositions of $\rho_{2}$. This can be extended to any density operator $\rho$, if it becomes $\rho_{2}$ by so-called purification, i. e., by constructing a bipartite state vector $|\Phi\rangle_{12}$ that implies the initial density operator as its second-subsystem reduced density operator.

One should note that, from the point of view of mathematical physics, this classification has an advantage over that of Cassinelli et al. ${ }^{4}$ (cf the Lemma above) consisting in the fact that the classifying entities and the details of the connection between them and the linearly-independent complete decompositions has a clear physical meaning in terms of the antilinear operator representation of $|\Phi\rangle_{12}$, and its polar factorization (cf section 2).

Remark 4: In Ref. 14, the approach of this section was indicated (without the antilinear operator representation) for finite-dimensional ranges. It was pointed out that this can lead to generating linearly-independent complete decomposition of states even at space-like separation. 


\section{THE SELECTIVE ASPECT OF REMOTE LINEARLY- INDEPENDENT COMPLETE STATE DECOMPOSI- TION: REMOTE STATE PREPARATION}

The selective or one-result sub-ensemble aspect of complete subsystem measurement that gives remote linearly-independent complete state decomposition was only implicitly given so far. Now we make it explicit. It is an immediate consequence of Theorem 1.

Theorem 2: Also the selective (or the one-result sub-ensemble) aspect, i. e., the remote preparations of pure states that are part of a linearlyindependent complete state decomposition, can be displayed in a commutative square diagram as below. The symbols on Diagram 2 have the following meaning.

$\mathbf{A}$ is the set of all state vectors $\left|e_{k}\right\rangle_{1}$ from $\mathcal{R}\left(\rho_{1}^{1 / 2}\right) \quad$ (equivalently, the set of all corresponding atomic events or ray projectors $\left|e_{k}\right\rangle_{1}\left\langle\left. e_{k}\right|_{1}\right)$. $\mathbf{B}$ is the set of all state vectors $\left|f_{n}\right\rangle_{2}$ from $\mathcal{R}\left(\rho_{2}^{1 / 2}\right)$. $\mathbf{C}$ is the set of all state vectors $\left|\chi_{n}\right\rangle_{1}$ from $\mathcal{R}\left(\rho_{1}\right)$. Finally, $\mathbf{D}$ is the set of all state vectors $\left|\phi_{k}\right\rangle_{2}$ from $\mathcal{R}\left(\rho_{2}\right)$.

The bijection $\mathbf{A} \longrightarrow \mathbf{B}$ comes about by application of the antiunitary correlation operator $U_{a}$, which is determined by the given bipartite state vector $|\Phi\rangle_{12}$. The inverse bijection $\mathbf{B} \longleftarrow \mathbf{A}$ is $U_{a}^{-1} . \quad \mathbf{A} \downarrow \mathbf{C}$ is $\quad p_{k}^{-1 / 2} \rho_{1}^{1 / 2}$. The inverse is $\mathbf{C} \uparrow \mathbf{A}=p_{k}^{1 / 2} \tilde{\rho}_{1}^{-1 / 2}$, where the tilde denotes the reducee to the range. $\mathbf{B} \downarrow \mathbf{D}$ is $p_{n}^{-1 / 2} \rho_{2}^{1 / 2}$. The inverse is $\mathbf{D} \uparrow \mathbf{B}=p_{n}^{1 / 2} \tilde{\rho}_{2}^{-1 / 2} . \quad \mathbf{C} \longrightarrow \mathbf{D}$ is $U_{a}$. The inverse bijection $\mathbf{C} \longleftarrow \mathbf{D}$ is $U_{a}^{-1}$.

The diagonal arrows, which have the physical meaning of remote state preparation, are the following. $\mathbf{A} \searrow \mathbf{D}$ is $p_{k}^{-1 / 2} \rho_{2}^{1 / 2} U_{a}$, or, equivalently, $p_{k}^{-1 / 2} A_{a}$. The inverse is $\mathbf{A} \nwarrow \mathbf{D}=p_{k}^{1 / 2} \tilde{\rho}_{1}^{-1 / 2} U_{a}^{-1}$.

$\mathbf{C} \swarrow \mathbf{B}$ is $p_{n}^{-1 / 2} \rho_{1}^{1 / 2} U_{a}^{-1}$, or, equivalently, $p_{n}^{-1 / 2} A_{a}^{\dagger}$. The inverse is $\mathbf{C} \nearrow \mathbf{B}=$ 
$p_{n}^{1 / 2} \tilde{\rho}_{2}^{-1 / 2} U_{a}$

\title{
Commutative Square Diagram 2. Remote Pure-State Preparation. (The Selective Aspect of Remote Linearly-Independent Complete State Decompositions)
}

\author{
$\mathbf{A} \equiv\left\{\right.$ all state vectors $\left.\left|e_{k}\right\rangle_{1} \in \mathcal{R}\left(\rho_{1}^{1 / 2}\right)\right\} \mathbf{B} \equiv\left\{\right.$ all state vectors $\left.\left|f_{n}\right\rangle_{2} \in \mathcal{R}\left(\rho_{2}^{1 / 2}\right)\right\}$
}

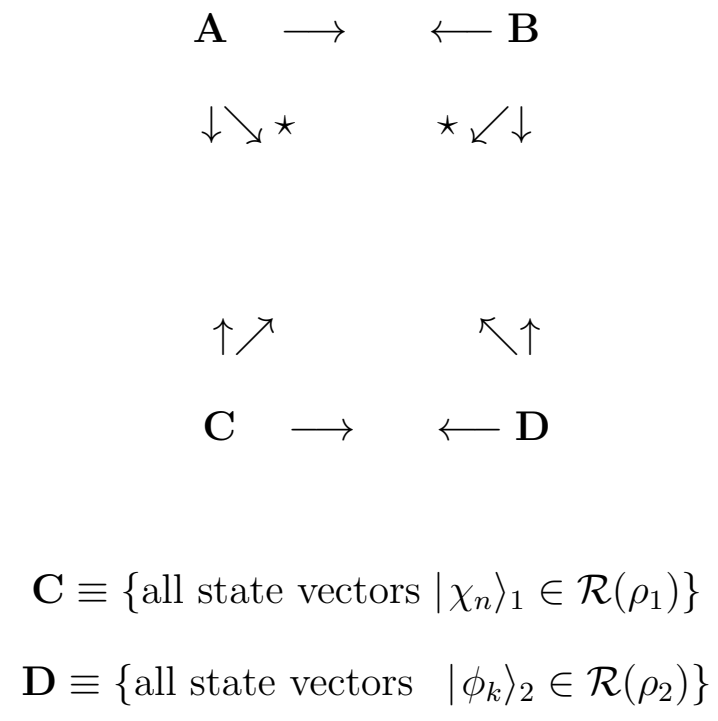

Caption. Each arrow goes from one of the sets (A,B,C,D) towards another. It stands for the corresponding bijection specified in Theorem 2. Oppositely oriented arrows denote mutually inverse bijections. The diagram is commutative, i. e., the successive bijections combine into the displayed corresponding one. For instance, taking the bijection $\mathbf{B} \downarrow \mathbf{D}$ after the bijection $\mathbf{A} \rightarrow \mathbf{B}$ gives the bijection $\mathbf{A} \searrow \mathbf{D}$, etc.

The imaginary vertical line cutting the square into two equally wide halves makes these completely symmetric (due to the symmetry between the two subsystems in $|\Phi\rangle_{12}$ ). 
* The downward diagonal bijections $\mathbf{A} \searrow \mathbf{D}$ and $\mathbf{C} \swarrow \mathbf{B}$ have the physical meaning of remote state preparations.

Theorem 2 completes previous work on remote preparation (or "steering", to use Schrödinger's term) begun by Schrödinger, ${ }^{1}$ and continued in Ref. 15. To make the completion more precise, the following theorem clarifies the issue.

Theorem 3: A) A state vector $|\phi\rangle_{2}$ is obtainable by remote preparation in $|\Phi\rangle_{12}$ if and only if $|\phi\rangle_{2} \in \mathcal{R}\left(\rho_{2}^{1 / 2}\right)$.

B) The set of all atomic events $|j\rangle_{1}\left\langle\left. j\right|_{1}\right.$ the occurrence of which in nearby-subsystem measurement in $|\Phi\rangle_{12}$ remotely prepares a given state vector $|\phi\rangle_{2}$ is (in terms of state vectors $|f\rangle_{1}$ and $|g\rangle_{1}$ ):

$$
\left\{|j\rangle_{1}\left\langle\left. j\right|_{1}: \mid j\right\rangle_{1}=\alpha|f\rangle_{1}+\beta|g\rangle_{1}\right\}
$$

where

$$
\begin{gathered}
|f\rangle_{1} \equiv U_{a}^{-1} \rho_{2}^{-1 / 2}|\phi\rangle_{2}, \\
|\alpha|>0, \quad|\alpha|^{2}+|\beta|^{2}=1,
\end{gathered}
$$

and

$$
|g\rangle_{1}=Q_{1}^{\perp}|g\rangle_{1}
$$

( $Q_{1}$ being the range projector of $\rho_{1}$, and $Q_{1}^{\perp}$ being its orthocomplementary projector, i. e., the null projector), otherwise $|g\rangle_{1}$ is arbitrary.

C) If the occurrence of the atomic event $|j\rangle_{1}\left\langle\left. j\right|_{1}\right.$ remotely prepares $|\phi\rangle_{2}$, then the probability of occurrence is proportional to $|\alpha|^{2}$ (cf (49a)). It is maximal if and only if $|j\rangle_{1}=|f\rangle_{1} \quad$ (cf (49b)).

D) If $|\phi\rangle_{2} \in \mathcal{R}\left(\rho_{2}\right) \quad(\operatorname{cf}(32))$, then $|f\rangle_{1} \in \mathcal{R}\left(\rho_{1}^{1 / 2}\right)$, where $|f\rangle_{1}$ is given by (49b). Thus, one has linearly-independent remote preparation in 
this case, where the maximal probability is the characteristic weight of $|\phi\rangle_{2}$ (cf Definition 3).

E) If $|\phi\rangle_{2} \in\left(\mathcal{R}\left(\rho_{2}^{1 / 2}\right)-\mathcal{R}\left(\rho_{2}\right)\right)$, where " -" denotes set-theoretical subtraction (of a subset), then $|f\rangle_{1} \in\left(\overline{\mathcal{R}}\left(\rho_{1}\right)-\mathcal{R}\left(\rho_{1}^{1 / 2}\right)\right)$, where $|f\rangle_{1}$ is given by (49b).

If the ranges of $\rho_{s}, s=1,2$ are finite dimensional, then the largest probability is always the characteristic weight corresponding to linearlyindependent remote preparation (cf (32)).

Proof of Theorem 3: A) The most general case of remote pure-state preparation in a bipartite state vector $|\Phi\rangle_{12}$ is given by (13d). Replacing $A_{a}$ by its polar-factorized form $\rho_{2}^{1 / 2} U_{a} Q_{1} \quad(\mathrm{cf}(9 \mathrm{~b})$ ), one can see that it is necessary that $|\phi\rangle_{2} \in \mathcal{R}\left(\rho_{2}^{1 / 2}\right)$. That this is also sufficient is obvious from the fact that $|\phi\rangle_{2}$ is obtained by remote preparation when the atomic event $|f\rangle_{1}\left\langle\left. f\right|_{1} \text { occurs, where } \mid f\right\rangle_{1}$ is given by (49b). (We again utilize the above polar-factorized form (9b) of $A_{a}$ in (13d).)

B) Since $|\phi\rangle_{2}=p^{-1 / 2} U_{a} \rho_{1}|j\rangle_{1}=p^{-1 / 2} U_{a} \rho_{1} Q_{1}|j\rangle_{1} \quad$ (cf (13d) and (9a)), where $p$ is the probability of occurrence, it is obvious that the occurrence of each of the atomic events $|j\rangle_{1}\left\langle\left. j\right|_{1} \quad \text { (cf (49a-d)) remotely prepares } \mid \phi\right\rangle_{2}$. On the other hand, (49a) with $|f\rangle_{1} \in \overline{\mathcal{R}}\left(\rho_{1}\right)$ is the general form of a state vector from $\mathcal{H}_{1}$, and $A_{a}=U_{a} \rho_{1}^{1 / 2} \quad(\mathrm{cf}(9 \mathrm{a}))$ is non-singular on $\overline{\mathcal{R}}\left(\rho_{1}\right)$, hence, it follows from (13d) that if $|j\rangle_{1}$ is not in the set (49a), then the occurrence of $|j\rangle_{1}\left\langle\left. j\right|_{1} \text { remotely prepares a state vector } \mid \phi^{\prime}\right\rangle_{2}$ that is distinct from $|\phi\rangle_{2}$.

C) Substituting in (13c) $\quad A_{a} Q_{1}$ instead of $A_{a}$ and $|j\rangle_{1}$ by its form in (49a), one obtains

$$
p=|\alpha|^{2}|| \rho_{1}^{1 / 2}|f\rangle_{1} \|^{2},
$$


which is independent of $|g\rangle_{2}$ (cf (49a)). Both claims in Theorem 3C are obvious from (50).

D) and E) The claims of Theorem 3D and 3E follow from the following set-theoretical insight. One has

$$
\overline{\mathcal{R}}\left(\rho_{1}\right)=\mathcal{R}\left(\rho_{1}^{1 / 2}\right)+\left(\overline{\mathcal{R}}\left(\rho_{1}\right)-\mathcal{R}\left(\rho_{1}^{1 / 2}\right)\right),
$$

and

$$
\mathcal{R}\left(\rho_{1}^{1 / 2}\right)=\mathcal{R}\left(\rho_{1}\right)+\left(\mathcal{R}\left(\rho_{1}^{1 / 2}\right)-\mathcal{R}\left(\rho_{1}\right)\right),
$$

where " +" denote the set-theoretical union of disjoint sets. The operator $\rho_{1}^{1 / 2}$ maps the first term on the rhs of (51) into the first term on the rhs of (52). This is seen from the fact that if $|j\rangle_{1} \in \mathcal{R}\left(\rho_{1}^{1 / 2}\right)$, then $\exists$ : $|k\rangle_{1}, \quad\left\langle\left. k\right|_{1} \mid k\right\rangle_{1}>0, \quad \rho_{1}^{1 / 2}|k\rangle_{1}=|j\rangle_{1}$. Then $\rho_{1}^{1 / 2}|j\rangle_{1}=\rho_{1}|k\rangle_{1} \in \mathcal{R}\left(\rho_{1}\right)$.

Actually, $\rho_{1}^{1 / 2}$ maps $\mathcal{R}\left(\rho_{1}^{1 / 2}\right)$ onto $\mathcal{R}\left(\rho_{1}\right)$. Namely, if $0 \neq|k\rangle_{1} \in$ $\mathcal{R}\left(\rho_{1}\right)$, then $\exists: 0 \neq|j\rangle_{1}$ such that $|k\rangle_{1}=\rho_{1}|j\rangle_{1}=\rho_{1}^{1 / 2}\left(\rho_{1}^{1 / 2}|j\rangle_{1}\right)$.

Finally, since $\rho_{1}^{1 / 2}$ maps the lhs of (51) onto the lhs of (52) in a nonsingular way, one easily concludes that this operator maps the second term on the rhs of (51) onto the second term on the rhs of (52).

\section{CONCLUDING REMARKS}

There is a very basic and elementary general claim: Every statement valid for all bipartite state vectors $|\Phi\rangle_{12} \in\left(\mathcal{H}_{1} \otimes \mathcal{H}_{2}\right) \quad$ is either symmetric in the two subsystems, or if not, then also the statement symmetrical to it is always valid. This comes from the essential symmetry between $\mathcal{H}_{1}$ and $\mathcal{H}_{2}$ (in spite of the fact that one has to use the two factor spaces in an ordered way).

The results of this article confirm the claim that at the very core of entanglement in any $|\Phi\rangle_{12}$ is the correlated subsystem picture (see section 2). It consists of statements that appear in symmetrical pairs: the two 
reducees $\tilde{\rho}_{1}$ and $\tilde{\rho}_{2}$ are symmetric (cf (10)) and so are the reducees of twin observables $\tilde{A}_{1}$ and $\tilde{A}_{2}$ (if one takes algebraic twin observables, i. e., ones with equal relevant spactra). The symmetry is in terms of the antiunitary correlation operator $U_{a}$ inherent in $|\Phi\rangle_{12}$, which establishes a sort of duality (like between kets and bras) between the closed ranges $\overline{\mathcal{R}}\left(\rho_{1}\right)$ and $\overline{\mathcal{R}}\left(\rho_{2}\right)$.

The correlation operator connects the orthogonal decompositions (or spectral forms) $\tilde{\rho}_{1}=\sum_{i} r_{i}|i\rangle_{1}\left\langle\left. i\right|_{1}, \quad \tilde{\rho}_{2}=\sum_{i} r_{i} \mid i\right\rangle_{2}\left\langle\left. i\right|_{2}, \quad\right.$ to which correspond the spectral forms of (physical) twin observables $\tilde{A}_{1}=\sum_{i} a_{i}|i\rangle_{1}\left\langle\left. i\right|_{1}\right.$ and $\tilde{A}_{2}=\sum_{i} a_{i}^{\prime}|i\rangle_{2}\left\langle\left. i\right|_{2}\right.$. (One should remember that the tilde denotes that the corresponding operator is reduced to the range of $\rho_{s}, s=1,2$. )

In the wider view, when also extended twin observables are taken into account, or, equivalently, when one considers generalized twin observables, which has been elaborated in this article, one treats the wider class of linearly independent subsystem state decompositions $\rho_{1}=\sum_{n} q_{n}\left|\chi_{n}\right\rangle_{1}\left\langle\left.\chi_{n}\right|_{1}\right.$ and $\rho_{2}=\sum_{k} p_{k}\left|\phi_{k}\right\rangle_{2}\left\langle\left.\phi_{k}\right|_{2}\right.$ along with the relevant generalized twin observables $A_{1}$ and $A_{2}$ the measurement of either of which gives rise to the mentioned state decomposition on the opposite subsystem (remote linearly-independent complete decomposition of state), but not to the decomposition on the same subsystem (except in the special case of proper twin observables). The full mathematical details and beauty of the generalized physical meaning of the correlated subsystem picture is expressed via the commutative diagrams.

If, following Schrödinger ${ }^{1}$ one tries to understand entanglement solely in terms of disentanglement, i. e., in terms of remote state decomposition, then one wonders what is left out from this article.

Restricting ourselves first to the (more important) complete state decompositions and pure-state preparation, the following is omitted. 
If the bipartite state vector has infinite entanglement, i. e., if the dimension of the two ranges of the respective reduced density operators is infinite, then even among the observables for which the basic commutation $\left[A_{s}, Q_{s}\right]=0, s=1,2$, is valid (range-compatible observables), those for which the eigenbases of the reducees $\tilde{A}_{s}, s=1,2$ are not entirely within $\mathcal{R}\left(\rho_{s}^{1 / 2}\right) s=1,2$ are left out from remote linearly-independent complete state decomposition. Further, equally for finite and for infinite entanglement, if at least one of the reduced density operators is singular, then the corresponding commutation $\left[A_{s}, Q_{s}\right]=0, s=1,2$ can be violated by some $A_{s}$, and the remote state decompositions caused by the measurement of such violating observables are also outside our treatment except in Theorem 3, which addresses the general case.

Considering only the non-selective aspect of remote influence, from the physical point of view it may not be clear why should one attach more importance to linearly-independent remote complete state decomposition than to the rest mentioned above. The answer lies in the selective aspect, when one considers remote linearly-independent pure-state preparation. Theorem $3 \mathrm{C}$ makes it clear that these are the nearby-subsystem occurrences that in measurement in $|\Phi\rangle_{12}$ have the highest probability. This fact singles them out in importance.

In Theorem 2 and Diagram 2 we have treated linearly-independent remote pure-state preparation as part of linearly-independent remote complete state decomposition. This is methodologically quite correct. But in view of the mentioned result in Theorem 3C, physically it is more satisfactory to reverse the roles of the non-selective and the selective aspects, and to consider the former as composed out of the latter. In other words, perhaps it is physically more correct to consider remote linearly-independent complete 
state decomposition as consisting of remote linearly-independent pure-state preparations. Then the physical importance of the latter is shared by the former.

One should point out that we have not considered incomplete remote linearly-independent state decomposition or remote linearly-independent mixedor-pure state preparation. This is much used in practice as a step towards complete state decomposition (towards pure-state preparation).

We may repeat the remark from the Introduction that in theoretical physics mathematics and physics are inextricably connected and the optimal form of the former, as a rule, gives physical insight, often in terms of new physical concepts. The correlated subsystem picture, by itself a mathematical concept, which has been further applied to disentanglement in this article, leads to insight into the structure of pure-state entanglement in terms of generalized (proper and extended) twin observables. In particular, linearly-independent remote pure-state preparation appears as the maximal-probability way of such a remote effect.

Finally, the largest-probability requirement in remote pure-state preparation leads to the conclusion (cf Theorem $3 \mathrm{C}$ ) that, from the physical point of view, in case of infinite-dimensional ranges of $\rho_{s}, s=1,2$, one should generalize "relevant" (for linearly-independent influence) observables by the weaker requirement of only range-compatible and detectably-complete ones (cf Definition 5). 


\section{Appendix A:}

Proof that

$$
\begin{gathered}
\left\{\left|\phi_{k}\right\rangle \notin \operatorname{span}\left\{\left|\phi_{1}\right\rangle, \ldots,\left|\phi_{(k-1)}\right\rangle,\left|\phi_{(k+1)}\right\rangle, \ldots,\left|\phi_{d}\right\rangle\right\},\right. \\
k=1,2, \ldots d ; d \in \mathbf{N}\} \Leftrightarrow \\
\left\{\forall k:\left|\phi_{k}\right\rangle \notin \operatorname{span}\left\{\left|\phi_{1}\right\rangle, \ldots,\left|\phi_{(k-1)}\right\rangle\right\}\right\},
\end{gathered}
$$

where $\mathbf{N}$ is the set of all natural numbers.

The first requirement on the set $\left\{\left|\phi_{i}\right\rangle: i=1, \ldots, d\right\}$ obviously implies the second one. To prove the inverse implication, we assume ab contrario that the first requirement is not valid, but the second is. Then there exists $k \in \mathbf{N}, \quad 1 \leq k \leq d$ such that

$$
\left|\phi_{k}\right\rangle=\sum_{i=1}^{(k-1)} \alpha_{i}\left|\phi_{i}\right\rangle+\sum_{j=(k+1)}^{d} \alpha_{j}\left|\phi_{j}\right\rangle
$$

all $\alpha_{i}$ and all $\alpha_{j}$ complex numbers. On account of the assumed validity of the second requirement in (A1.1), not all $\alpha_{j}$ can be zero. We define $\bar{j} \equiv \max \left\{j: \alpha_{j} \neq 0\right\}$. Then (A1.2) implies

$$
\left|\phi_{\bar{j}}\right\rangle=\alpha_{\bar{j}}^{-1}\left(\left|\phi_{k}\right\rangle-\sum_{i=1}^{(k-1)} \alpha_{i}\left|\phi_{i}\right\rangle-\sum_{j=(k+1)}^{(\bar{j}-1)} \alpha_{j}\left|\phi_{j}\right\rangle\right)
$$

in contradiction to the assumed validity if the second requirement.

\section{Appendix B:}

Proof (of Corollary 1) that every finite subset $\left\{\left|\phi_{1}\right\rangle, \ldots,\left|\phi_{k}\right\rangle\right\}$ of a linearly-independent set (finite or infinite) is a linearly-independent basis in the $k$-dimensional subspace $\mathcal{S}_{k}$ that it spans. First we prove the claimed dimensionality of the span. 
Total induction. We assume that the dimensionality claim is true for $(n-1)<k: \quad \operatorname{span}\left\{\left|\phi_{1}\right\rangle, \ldots,\left|\phi_{(n-1)}\right\rangle\right\}=\mathcal{S}_{(n-1)}$. Let $\left|\phi_{n}\right\rangle$ be linearlyindependent of the mentioned preceding state vectors. Let $P$ project onto $\mathcal{S}_{(n-1)}$. One has

$$
\left|\phi_{n}\right\rangle=P\left|\phi_{n}\right\rangle+P^{\perp}\left|\phi_{n}\right\rangle,
$$

where $P^{\perp} \equiv(1-P)$, and $P^{\perp}\left|\phi_{n}\right\rangle$ cannot be zero (cf Definition 1). We define $\left|f_{n}\right\rangle \equiv c P^{\perp}\left|\phi_{n}\right\rangle$, where $c$ is a normalization constant, and

$$
\mathcal{S}_{n} \equiv \operatorname{span}\left\{\mathcal{S}_{(n-1)},\left|f_{n}\right\rangle\right\}
$$

is a subspace of $n$ dimensions. Since $\left|f_{n}\right\rangle=c\left(\left|\phi_{n}\right\rangle-P\left|\phi_{n}\right\rangle\right)$, $\mathcal{S}_{n} \subset \operatorname{span}\left\{\left|\phi_{1}\right\rangle, \ldots,\left|\phi_{n}\right\rangle\right\}$. It is obvious from (A2.1) that $\left|\phi_{n}\right\rangle \in \mathcal{S}_{n}$. Hence, $\operatorname{span}\left\{\left|\phi_{1}\right\rangle, \ldots,\left|\phi_{n}\right\rangle\right\} \subset \mathcal{S}_{n}$, and, finally, $S_{n}=\operatorname{span}\left\{\left|\phi_{1}\right\rangle, \ldots,\left|\phi_{n}\right\rangle\right\}$.

Since the claim that the span is a subspace of that many dimension as the number of linearly-independent state vectors is true for $n=1$, total induction implies that it is true for any $n \leq k$.

The uniqueness of the expansion follows from Corollary 3 is one takes an arbitrary probability distribution $\left\{p_{i}: i=1,2, \ldots, k ; p_{i}>0 ; \sum_{i=1}^{k} p_{i}=1\right\}$ and one defines $\rho \equiv \sum_{i=1}^{k} p_{i}\left|\phi_{i}\right\rangle\left\langle\phi_{i}\right|$.

Appendix C: Proof of Corollary 3. We show that assuming (27), each element $|\chi\rangle$ from the range $\mathcal{R}\left(\rho^{1 / 2}\right)$ can be expanded in the linearlyindependent sequence $\left\{\left|\phi_{i}\right\rangle: i=1,2, \ldots, d \leq \infty\right\}$.

Let

$$
\tilde{\rho}^{-1 / 2}|\chi\rangle=\sum_{i} \beta_{i}\left|e_{i}\right\rangle .
$$

Applying the continuous operator $\rho^{1 / 2}$, one obtains (cf Lemma B)):

$$
|\chi\rangle=\sum_{i} \beta_{i} \rho^{1 / 2}\left|e_{i}\right\rangle=\sum_{i} \beta_{i} p_{i}^{1 / 2}\left|\phi_{i}\right\rangle
$$




$$
|\chi\rangle=\sum_{i} \alpha_{i}\left|\phi_{i}\right\rangle
$$

where $\forall i: \quad \alpha_{i}=\beta_{i} p_{i}^{1 / 2}$. On account of (A3.1) and (28), one has

$$
\forall i: \quad \alpha_{i}=p_{i}^{1 / 2}\left[\left(\left\langle e_{i}\right| \tilde{\rho}^{-1 / 2}\right)|\chi\rangle\right]
$$

Substituting $\left\langle e_{i}\right|$ from (30), the last relation enables us to rewrite (A3.2) as follows:

$$
|\chi\rangle=\sum_{i} p_{i}\left[\left(\left\langle\phi_{i}\right| \tilde{\rho}^{-1}\right)|\chi\rangle\right]\left|\phi_{i}\right\rangle .
$$

Finally, the uniqueness of the expansion (A3.3) is easily proved by bringing the opposite assumption into contradiction with the definition of linear independence (cf Definition 1).

${ }^{1}$ E. Schrödinger, Proc. Cambridge Phil. Soc. 31, 555 (1935).

${ }^{2}$ F. Herbut and M. Vujičić, Ann. Phys. (NY) 96, 382 (1976).

${ }^{3}$ F. Herbut and M. Vujičić, in Proceedings of the International School of Physics "Enrico Fermi", Course IL, ed. B. d'Espagnat (Academic Press, London, 1971), pp. 316-329.

${ }^{4}$ G. Cassinelli, E. De Vito, and A. Levrero, J. Math. Analys. and Appl. 210, 472 (1997).

${ }^{5}$ I. M. Gel'fand and N. Ya. Vilenkin, Generalized Functions, Vol. 4 (Academic Press, New York, 1964).

${ }^{6}$ J. M. Jauch, Foundations of Quantum Mechanics, pp. 175-178 (AddisonWesley, Reading, Mass.,1968).

${ }^{7}$ F. Herbut and M. Vujičić, J. Math. Phys. 8, 1345 (1967).

${ }^{8}$ M. Vujičić and F. Herbut, J. Math. Phys. 25, 2253 (1984).

${ }^{9} \mathrm{~F}$. Herbut, e-print quant-ph/0403101.

${ }^{10}$ F. Herbut, Physical Review A 66, 052321-1-6 (2002). 
${ }^{11}$ N. Hadjisavvas, Lett. Math. Phys. 5, 327 (1981).

${ }^{12}$ I. C. Gohberg and M. G. Krein, Introduction to the Theory of Linear Nonselfadjoint Operators, Translations of Mathematical Monographs, Vol. 18 (AMS, Providence, R. I., 1969).

${ }^{13}$ P. Busch, P. J. Lahti, and P. Mittelstaedt, The Quantum Theory of Measurement, Lecture Notes in Physics M2 (Springer-Verlag, Berlin, 1991).

${ }^{14}$ L. P. Hughston, R. Jozsa, and W. K. Wootters, Phys. Lett. A 183, 14 (1993).

${ }^{15}$ F. Herbut and M. Vujičić, J. Phys. A 20, 5555 (1987). 